\title{
La didattica a distanza alla prova della primaria
}

Esperimenti e riflessioni sul suo valore formativo nel primo ciclo d'istruzione di Cristina Ambrogetti

Dic 17, 2020 | Didattica a distanza, In evidenza, Pensare la didattica $|\underline{0}|$

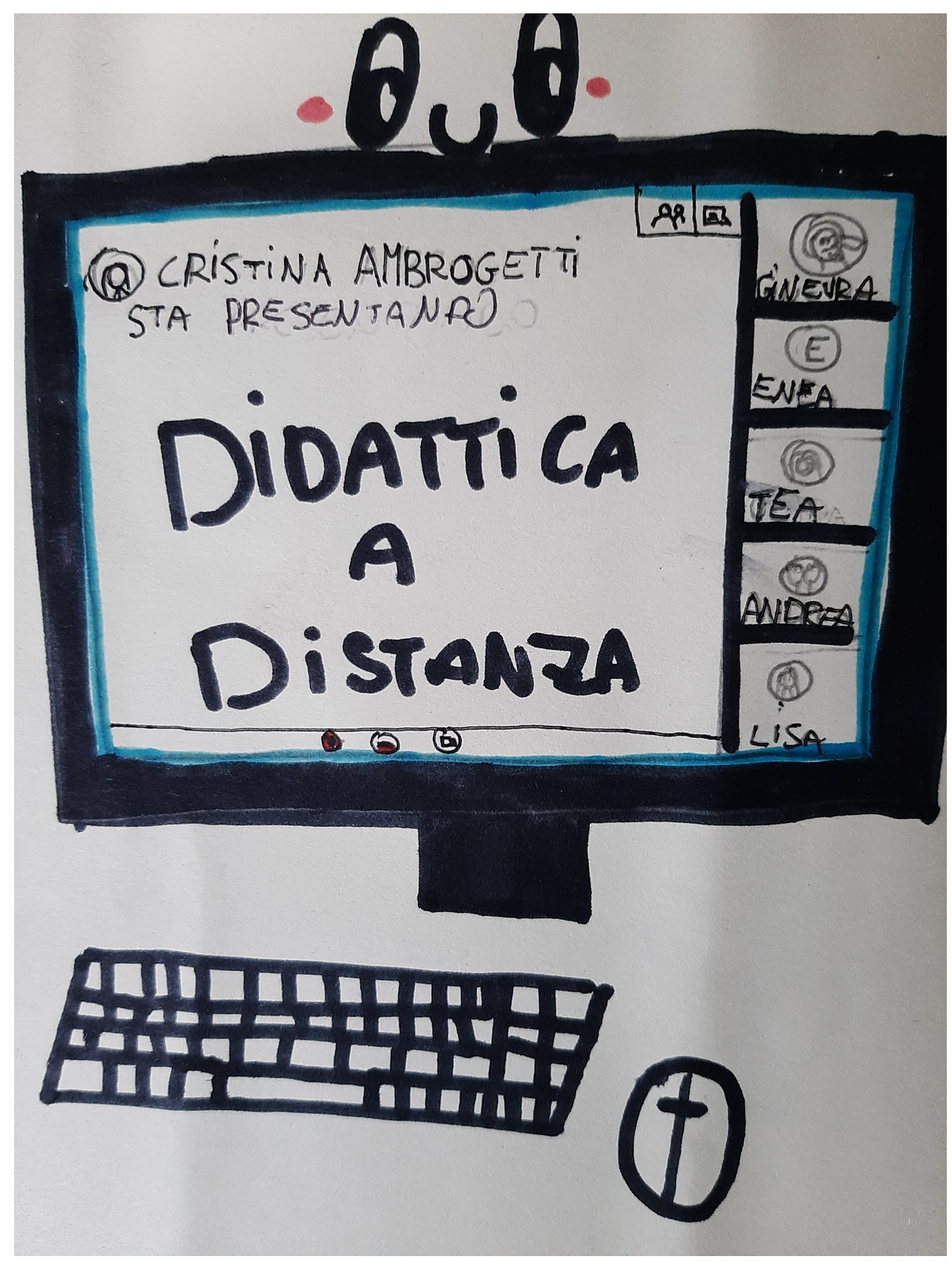


Ciò che qui si racconta, l'esperienza didattica del processo inteso come un $j$ 'accuse alla Didattica a Distanza scritto e interpretato sotto forma di rappresentazione teatrale dagli alunni della classe quinta della Scuola Primaria di Castiglione di Ravenna, è l'atto conclusivo (e catartico) di un processo sviluppatosi nel senso etimologico di processus «avanzamento, progresso» verso la conoscenza e la comprensione di un fenomeno e delle sue conseguenze più immediate per $\mathrm{i}$ bambini: la pandemia di Covid-19 e la chiusura

Riflettere sul senso profondo della didattica, in un tempo sospeso e complesso come quello che stiamo vivendo, riporta alla memoria l'episodio con il quale Marc Bloch apre Apologia della Storia, una domanda posta da un figlio al padre in un momento storico che preludeva al secondo conflitto mondiale: «Papà, spiegami a che serve la storia». Il proposito che quella domanda improvvisa $\mathrm{e}$ imprevista meritasse una risposta all'altezza delle aspettative, costituisce per Bloch l'impulso a scrivere sulla legittimità della storia.

Da più parti ho sentito ripetere che la scuola o è in presenza, o altrimenti non è scuola, poiché il processo educativo sarebbe annientato da una "non-didattica a distanza". Quindi: a che serve la didattica a distanza? Parallelamente, molti pedagogisti, dirigenti scolastici e insegnanti hanno visto nell'emergenza Coronavirus l'occasione per fare delle competenze di comunicazione virtuale un elemento di innovazione, un mezzo per aprire un varco verso lo spazio della relazione e della vicinanza, a dispetto del distanziamento imposto dalla situazione di crisi. L'educazione attiva è possibile se e in quanto l'allievo è attivo, e può imparare anche dagli imprevisti.

Nella scuola in cui insegno, gli alunni, giunti al loro ultimo anno nella Scuola Primaria, hanno sperimentato insieme agli insegnanti un nuovo modo di fare scuola e contemporaneamente hanno costruito strumenti per conoscere e comprendere il presente, senza subirlo o esserne travolti, ma raccogliendo tracce per poterlo affrontare con lucidità.

\section{Dalla didattica dell'emergenza alla didattica della vicinanza}

Il DL 23 febbraio 2020 n.6, contenente le Misure urgenti in materia di contenimento e gestione dell'emergenza epidemiologica da COVID-19, ha decretato la «sospensione dei servizi educativi dell'infanzia e delle scuole di ogni ordine e grado, nonché della frequenza delle attività scolastiche e di formazione superiore, compresa quella universitaria, salvo le attività formative svolte a distanza».

Pochi giorni dopo, il DPCM 8 marzo 2020 confermava l'espressione "Didattica a Distanza" (DaD) per indicare le attività che gli insegnanti e gli studenti, impossibilitati a frequentare in presenza le aule scolastiche, avrebbero svolto da quel momento utilizzando attrezzature informatiche e connessioni da remoto. Nelle due settimane intercorse fra le due norme si è un acceso su più fronti un partecipato dibattito, con mozioni a favore e mozioni contro.

È bene ricordare come parte attiva per la strutturazione del palinsesto per l'avvio della Didattica a Distanza siano stati moltissimi genitori, a cominciare dai rappresentanti di classe i quali, ancora una volta a dispetto dei limiti imposti dalla ridotta mobilità, hanno contribuito a curare il passaggio delle informazioni e a gestire le comunicazioni, creando un vero e proprio sistema di auto mutuo aiuto, destreggiandosi tra telefono, Whatsapp, e-mail per affiancare chi, non particolarmente avvezzo alla tecnologia, si trovava in difficoltà con account, piattaforme e link per connettersi alle videolezioni. Come nel perenne susseguirsi del moto marino per ogni onda che guadagna la riva c'è quella successiva che sopraggiunge, così le scuole, a fronte di tempi di chiusura che diventavano "indeterminati", hanno fatto appello nuovamente alle proprie risorse professionali per ragionare sull'offerta formativa, riorganizzando i curricoli nella cornice della condivisione collegiale. 
Anche nella mia scuola, via via che le attività a distanza e le videolezioni si susseguivano in un calendario di appuntamenti giornalieri, ci si rendeva conto di quanto fosse diventata bella l'attesa di quegli incontri: una nicchia sicura per praticare lo spazio della relazione, un tempo per arrivare da compagni al tramonto del giorno e, da compagni, condividere pensieri e notizie, domandare e cercare risposte.

All'inizio è stato un gioco di scrittura: realizzare un racconto collettivo di fantascienza "a staffetta". Si voleva raccontare la storia di un gruppo di giovani scienziati, chiusi da tempo nel loro laboratorio, che decidono di scrivere un diario da lasciare come testimonianza ai posteri. Il racconto inizia così:

«Diario dell'ANNO SOLARE 202020, sul Pianeta Terra stanno succedendo cose mai accadute: un intero continente sta bruciando, i ghiacci si stanno sciogliendo e ora questo... un VIRUS SCONOSCIUTO che silenziosamente si diffonde tra le Nazioni.

Io e la mia squadra di scienziati stiamo cercando di fermare il contagio prima che sia troppo tardi. Ma per sicurezza abbiamo deciso di scrivere tutta la storia in questo diario da lasciare ai posteri per il futuro.

Tutto è iniziato così. In una nazione chiamata Xyna, un giorno al mercato un signore si avvicinò ad una bancarella per comprare del cibo. La signora che stava alle vendite, una donna che tossiva e starnutiva molto rumorosamente, consigliò al signore di comprare...»

Che cosa succederà dopo? Chi vuole continuare la storia? Chi si offre di ricevere il testimone, deve proseguire il racconto aggiungendo 3 frasi e leggerle ai compagni alla fine della videolezione successiva.

Siamo andati avanti così. Inanellando una frase dopo l'altra, il racconto è passato di mano in mano e si è arricchito di giorno in giorno. Come i giovani scienziati del racconto, anche i bambini avevano uno scopo affascinante e si impegnavano con una finalità precisa e concreta. Potremmo dire un "compito autentico".

\section{Dal dibattito al processo}

Lo sviluppo quotidiano del racconto-diario ha sollecitato l'attenzione verso la situazione presente, annotandone l'evoluzione con spirito di ricerca. I frammenti delle informazioni raccolte, delle notizie sentite alla televisione o dei discorsi fatti dagli adulti di casa, venivano sottoposti all'attenzione dei compagni e dell'insegnante e insieme analizzati, approfonditi, ridimensionati o smentiti. Ne è nato un "giro di parola" quotidiano che è via via cresciuto diventando uno scambio di opinioni che ha dato origine a un vero e proprio dibattito. A pochissime settimane dalla fine della scuola, abbiamo ritenuto che tutto ciò che ci eravamo detti meritasse di essere trascritto e lo abbiamo organizzato nella forma di un ipotetico "Processo alla Didattica a Distanza", in modo che ne rimanesse memoria, sia per gli alunni direttamente coinvolti, sia per gli altri compagni. Ognuno è stato libero di scegliere se ritenersi avvocato dell'accusa (A) o avvocato della difesa (D).

Il risultato appare come una finestra aperta sulla realtà vissuta dai bambini, testimoni a volte diretti e autentici, altre volte inconsapevolmente condizionati dalle loro famiglie e dai media. Si susseguono così riflessioni ibride, indice di quello che i bambini pensano, ma anche di ciò che assorbono dal contesto familiare e soprattutto comunicativo dei media. Si notano registri diversi, 
pensieri ora adulti ora infantili, incertezze e contraddizioni che si intrecciano in un insieme complesso che bene rappresenta l'esposizione informativa ed esperienziale ad una situazione difficile, nuova e confusa per tutti. Certamente appare positivo aver lasciato sgorgare ogni cosa perché trovasse una più razionale sistematizzazione.

È stata poi scelta la data corrispondente all'ultimo giorno di scuola per incontrarsi, all'aperto e distanziati, in un grande parco pubblico e animare il processo pubblicamente dal vivo, con i gesti e con le voci, davanti ai genitori e agli insegnanti.

A: Io accuso la Didattica a Distanza perché ci ha fatto crescere troppo in fretta e ci ha derubato di una parte della nostra infanzia. Spesso dovevamo stare a casa da soli a seguire le lezioni perché i nostri genitori andavano a lavorare e siamo stati costretti a sostenere degli impegni da adulti.

D: La Didattica a Distanza ci ha resi più responsabili e maturi. Non ci ha rubato niente, anzi, ci ha arricchiti: ora sappiamo usare meglio il computer, sappiamo usare Power Point e fare presentazioni durante le video lezioni, abbiamo imparato a fare ricerche a gruppi in videochiamata.

A: Io accuso la Didattica a Distanza perché ha messo in difficoltà molte famiglie, soprattutto quelle con più figli, perché è difficile gestire le consegne dei compiti e gli orari delle lezioni che spesso coincidono tra loro.

D: È stato difficile perché non eravamo abituati, ma col passare del tempo, piano piano abbiamo trovato il modo di organizzarci al meglio.

A: Io accuso la Didattica a Distanza per avere stravolto la nostra vita quotidiana!

D: Abbiamo dovuto abbandonare le nostre abitudini, ma chi lo dice che il cambiamento è stato solo in peggio? Scommetto che a tutti piace dormire un po' di più alla mattina.

A: Io accuso la didattica a distanza perché a scuola segniamo i compiti sui diari, a casa invece dobbiamo entrare su classroom per leggerli e se la connessione non funziona bene non possiamo vederli e questo mi crea ansia;

D: Il problema si risolve organizzandosi per tempo: quando si accede a classroom basta prendere nota di tutti i compiti presenti e non solo di quelli a breve scadenza.

A: Io accuso la Didattica a Distanza perché ci ha tappato la bocca! Ci ha impedito di chiacchierare con i nostri compagni e questo dover tenere i microfoni disattivati mi ha scossa...

A: ...e io accuso la Didattica a Distanza perché non ci ha permesso di fare ricreazione insieme ai compagni e di parlare tra di noi!

D: Noi ci incontriamo su Meet sempre in anticipo rispetto all'inizio delle lezioni e abbiamo tutto il tempo per chiacchierare.

A: Io accuso la Didattica a Distanza perché ci fa perdere tempo: spesso salta la connessione internet e ogni volta bisogna rifare il percorso per entrare su classroom...

A: ...e io l'accuso perché con il segnale internet poco stabile non riusciamo ad ascoltare tutta la lezione: altro che Didattica! Qui si lede il diritto allo studio! 
D: Non prendiamoci in giro! Perché forse quando eravamo in classe stavamo sempre attenti? È troppo facile dare la colpa di tutto alla Didattica a Distanza. Anche in classe eravamo distratti e i maestri dovevano richiamarci a seguire le lezioni con più attenzione.

A: Io accuso la Didattica a Distanza di averci dato "una scuola a metà" perché le ore di lezione a distanza sono state di meno di quelle che avremmo fatto in presenza a scuola...

A: ...e io accuso la Didattica a Distanza perché ci ha tolto la ricreazione che era importante per prenderci una pausa per spezzare le ore di lezione.

D: Questo è vero solo in apparenza. Ancora una volta la Didattica a Distanza non toglie nulla, ma ci invita a vivere il tempo con responsabilità e attenzione. La Didattica a distanza ci invita a migliorarci.

A: Io accuso la Didattica a Distanza perché non possiamo vedere i nostri compagni di classe.

D: È ovvio che vedersi in video non è come vedersi dal vivo, ma è anche vero che molti di noi tengono la videocamera spenta. Se hanno inventato le videocamere, sfruttiamole, accendiamole...

D: ...e aggiungo che per tutto il tempo in cui eravamo costretti a stare chiusi in casa perché a causa della pandemia non si poteva uscire, la Didattica a Distanza ci ha dato il sollievo di poter vedere per qualche ora al giorno i nostri amici.

A: Io accuso la Didattica a Distanza perché per connetterci e partecipare alla video lezione dobbiamo usare dei GIGA che sono a pagamento, invece la Costituzione stabilisce che la scuola deve essere gratuita e accessibile a tutti. Le famiglie si sono accollate un costo.

D: In questi mesi di scuola chiusa ci siamo risparmiati il costo della mensa. Inoltre del costo della connessione non è direttamente responsabile la Didattica a Distanza, ma le compagnie telefoniche... casomai sono loro da processare!

A: Io accuso la Didattica a Distanza di avere favorito i "furbetti", quelli che, se non sapevano rispondere durante un'interrogazione, uscivano dalla lezione facendo finta di avere problemi di connessione. Se fossimo stati a scuola non sarebbero mica potuti uscire dall'aula!

D: Se uno voleva fare il furbetto, trovava il modo anche a scuola, ad esempio dicendo di avere lasciato il quaderno a casa...

A: Io accuso la Didattica a Distanza di avere rovinato il finale della quinta elementare: per quattro anni abbiamo accompagnato al cancello con applausi e striscioni colorati i nostri compagni dell'ultimo anno e ora che finalmente era arrivato il nostro momento, tutto è andato in fumo per colpa della Didattica a Distanza.

D: Riflettiamo un attimo: è vero che non abbiamo avuto l'ultimo giorno di scuola dei nostri sogni, ma abbiamo avuto altri momenti speciali. Senza la Didattica a Distanza non avremmo mai incontrato in videolezione Roberto Piumini per parlare di poesia!

A: Io accuso la Didattica a Distanza di averci privati degli spazi che da sempre rappresentano la scuola: i banchi, la lavagna, la scrivania della maestra e quell'atmosfera che si respira e si vive solo all'interno di un'aula. 
D: Stare davanti a un computer non è come stare a scuola, ma viviamo gli spazi in modo diverso: la casa, con un po' di immaginazione si può trasformare: la scrivania o il tavolo diventano il banco, lo schermo del computer diventa la lavagna su cui scrivere e proiettare materiale.... Su amici, usiamo l'immaginazione e la fantasia!

A: Io accuso la didattica a distanza perché ci ha privato dei viaggi di istruzione che ci facevano divertire ed imparare allo stesso tempo.

D: Un attimo... se ci pensiamo bene le gite virtuali grazie ai siti dei musei non sono mancate, abbiamo visitato addirittura il British Museum!

Al termine del dibattimento simulato, il verdetto è stato proclamato dal pedagogista Andrea Canevaro, professore emerito dell'Università di Bologna, ed è risultato favorevole alla Didattica a Distanza, che è stata assolta, ma con l'obbligo di eseguire lavori utili: dovrà «collaborare con la didattica in presenza per migliorare le attività scolastiche perché non siano unicamente lezioni, ma anche laboratori, uscite, esperienze. Tutte attività che permettono di riprendere ritmi compatibili con una vita sociale piena e fatta di incontri». (A. Canevaro).

\section{Fare memoria e fare comunità}

A conclusione di questa esperienza, pensiamo che la differenza che esiste fra la scuola in presenza e quella a distanza sia simile a quella che si usa nel campo della profumeria per distinguere il parfum dall'eau de toilette: il dato che li caratterizza è la diversa concentrazione di alcool e di oli essenziali. Le attività che si svolgono a distanza devono, come il parfum essere più intense e durature. La loro credibilità dipende dalla capacità di "persistere" e riconduce al significato di "fermarsi". Fermarsi ad aspettare, fermarsi ad ascoltare. Non possono essere gestite semplicemente come se fossero la trasposizione in uno spazio virtuale dell'attività che si sarebbe svolta nell'ambiente fisico. E non vanno nemmeno trattate come una sintesi né come un surrogato.

Ispirarsi all'abilità e alla perizia artigianale di un artisan parfumeur può servire: la didattica che si fa quando le scuole sono chiuse è oggettivamente diversa dalla didattica proposta quando le scuole sono aperte, ma gli alunni, i loro genitori e gli insegnanti devono poter riconoscere che la "fragranza" è la medesima. Occorre un'attenta consapevolezza in tutte le fasi, per non perdere il dato originale, per non alterarlo, rendendolo irriconoscibile o stomachevole.

Ma la scuola in questo tempo complesso ha davanti a sé un'occasione importante per fare memoria. Per non resettare ogni cosa e chiudersi in futuro in un'aula come se nulla fosse stato o fosse solo un capitolo chiuso da dimenticare. La scuola, dopo il Coronavirus, dovrà affrontare una nuova sfida per nutrire e dare continuità alle competenze che i ragazzi hanno iniziato ad acquisire insieme ai loro insegnanti, per organizzarle nell'ottica di un apprendimento etico. Il diario e il dibattimento sono due strumenti scelti per facilitare la raccolta di informazioni e la riflessione personale mentre si è immersi nel flusso degli eventi in divenire. Marc Bloch sosteneva che fosse fatica inutile cercare di comprendere il passato se non si sa nulla del presente. Possiamo far diventare una virtù la necessità di insegnare sapendo che la scuola al tempo del Coronavirus non è e non potrà essere come la scuola prima del Coronavirus. Più in generale, non si potrà prescindere dalle scelte che si stanno delineando a livello locale e a livello globale per spiegare i nuovi assetti attorno ai quali si modelleranno per gli anni a venire i comportamenti individuali, le abitudini sociali, gli scenari economici e politici, la ricerca scientifica e le espressioni artistiche. Possiamo abituare i ragazzi a servirsi della comprensione dei periodi più vicini a loro, che costituiscono zone di relativa chiarezza, per fare memoria e fare comunità. In che modo? 


\section{Non solo debate}

Le generazioni di insegnanti che attualmente lavora nella scuola, è cresciuta ascoltando i racconti di genitori e nonni che narravano un tempo da loro vissuto direttamente, anni che hanno determinato una frattura fra un "prima" e un "dopo" per eccellenza: la guerra. E sicuramente, o per curiosità personale o per condurre piccole ricerche scolastiche, gli insegnanti di oggi quando erano piccoli studenti hanno posto delle domande: hanno intervistato i familiari raccogliendo testimonianze di vita vissuta all'interno del contesto della comunità di appartenenza. Tante storie individuali come frammenti che si contestualizzano nel più ampio orizzonte della Storia. Un orizzonte che può essere compreso nella sua reale ampiezza e profondità solo a distanza di tempo dagli eventi.

In questi giorni sta facendo il giro dei telegiornali e dei social la singolare campagna di sensibilizzazione rivolta ai giovani, realizzata dal Governo tedesco, sui comportamenti da tenere per contrastare la diffusione del Covid-19. Si tratta di un breve video girato come fosse un documentario storico, ma ambientato in un futuro immaginario. Un anziano signore racconta di quando, ventiduenne studente di ingegneria, nell'inverno del 2020 mentre imperversava la seconda ondata di Covid-19, si era trovato suo malgrado nelle mani il destino dell'intera nazione e, insieme a tanti altri ragazzi come lui, aveva saputo fare la cosa giusta: «Assolutamente niente. Siamo stati pigri come procioni. Abbiamo combattuto il coronavirus restando in casa». Lo spot tedesco è ironico, ma evidenzia in modo esemplare un aspetto davvero interessante: ciò che stiamo vivendo come presente e quotidiano, ha una portata storica significativa per il mondo intero che vive un momento di difficoltà contemporaneamente su scala planetaria. Ed è a mio parere altrettanto significativo che il video sia strutturato come documentario-intervista.

«Comprendere non sottintende affatto un atteggiamento di passività» diceva ancora Marc Bloch. E l'intervista come strumento didattico può essere un mezzo privilegiato di apprendimento attivo e cooperativo per la centralità del ruolo svolto dai ragazzi in tutte le fasi e per il suo valore di documentazione. L'intervista deve avere uno scopo che deve essere chiaro a chi prende parte al colloquio. Devono essere chiari e riconoscibili anche i ruoli dei partecipanti: intervistatore e intervistato hanno ruoli distinti. Nell'intervista, le persone coinvolte interagiscono in una situazione strutturata che prevede uno scambio di domande e di risposte e presuppone che l'intervistatore abbia in ogni momento ben chiaro a che punto si è arrivati e qual è stato il percorso seguito.

Anche l'intervista, condotta in modo mirato e coerente, è uno strumento conoscitivo e di indagine in grado di centrare l'obiettivo, di mediare un canale di dialogo fra presente e passato, o fra vicino e lontano per guidare i ragazzi ad avere una visione più ampia degli avvenimenti trascorsi attraverso una sistematica raccolta delle testimonianze che costituiscono un serbatoio per la memoria della comunità. Se sapremo agire facendo tesoro di ciò che viviamo in questo tempo di crisi e costruiremo una comunità consapevole della propria memoria, eviteremo di raccogliere macerie.

\section{Bibliografia}

- R. Biasi Neri, A. Canevaro, Programmazione e difficoltà scolastiche, Mondadori, Milano 1979

- M. Bloch, Apologia della storia o mestiere di storico, PBE Einaudi, Torino 1969

- A. Canevaro, Le logiche del confine e del sentiero. Una pedagogia dell'inclusione (per tutti, disabili inclusi), Erickson, Trento 2006

- A. Canevaro, A. Chieregatti, La relazione di aiuto. L'incontro con l'altro nelle professioni educative, Carocci, Roma 1999

- S.Kanizsa, Che ne prnsi? L'intervista nella pratica didattica, la Nuova Italia scientifica, Roma 1993 
- F. Lorenzoni, I bambini pensano grande. Cronaca di una avventura pedagogica, Sellerio Editore, Palermo 2014

- N.Revelli, Il mondo dei vinti, Einaudi, Torino 1977 